\title{
Re-irradiation with interstitial brachytherapy in uterine cancer patients with vaginal recurrence after post-operative pelvic irradiation
}

\author{
Keiko Nemoto Murofushi, MD, PhD',2, Reiko Tanaka, MD!, Ayako Ohkawa, MD, PhD!, Haruko Numajiri, MD, PhD!, \\ Shota Minakami, MD², Toshiyuki Okumura, MD, PhD', Hideyuki Sakurai, MD, PhD' \\ IFaculty of Medicine, Department of Radiation Oncology and Proton Medical Research Center, University of Tsukuba, Tsukuba, Ibaraki, Japan, \\ 2Division of Radiation Oncology, Department of Radiology, Tokyo Metropolitan Cancer and Infectious Diseases Center Komagome Hospital, \\ Bunkyo-ku, Tokyo, Japan
}

\begin{abstract}
Purpose: This study aimed to evaluate the outcomes and dose-volume parameters of re-irradiation with interstitial brachytherapy (ISBT) in uterine cancer patients with vaginal recurrence after post-operative pelvic irradiation.

Material and methods: We evaluated six uterine cancer patients who received re-irradiation with ISBT between January, 2012 and December, 2016. As an initial treatment, all patients received post-operative whole pelvic irradiation of 45-50.4 Gy in 25-28 fractions. For vaginal recurrence, all patients were treated with ISBT alone at a dose of 38-42 Gy in 6-7 fractions for clinical target volumes (CTVs) for 3-4 days.

Results: Post-operative pelvic irradiation was delivered to five and one patients, using a three-dimensional conformal technique and intensity-modulated radiotherapy, respectively. Median duration from surgery to vaginal recurrence was 25.7 months. Median tumor size just before ISBT was $3.3 \mathrm{~cm}$. Median time from completion of pelvic irradiation to ISBT initiation was 24.1 months. Mean doses per fraction of ISBT for CTV $\mathrm{D}_{90}$ (the minimum dose received by $90 \%$ of $\mathrm{CTV})$ and minimum dose received by $2 \mathrm{cc}\left(\mathrm{D}_{2 \mathrm{cc}}\right)$ for the bladder and for rectum were $6.1 \mathrm{~Gy}, 4.4 \mathrm{~Gy}$, and 3.8 Gy, respectively. Mean total equivalent dose in $2 \mathrm{~Gy}$ fractions $\left(\mathrm{EQD}_{2}\right)$, including external beam radiotherapy and ISBT, for $\mathrm{D}_{2 \mathrm{cc}}$ for the bladder, sigmoid, and rectum were $92.1 \mathrm{~Gy}, 50.4 \mathrm{~Gy}$, and 81.6 Gy, respectively. Median follow-up duration was 53.3 months. Local recurrence was observed in two patients, and four of the six patients were alive. Grade 2 late rectal complications occurred in two patients, and no late grade $\geq 3$ complications were observed in four alive patients.

Conclusions: Re-irradiation with ISBT may be an effective treatment strategy for gynecological cancer patients with vaginal recurrence after post-operative pelvic irradiation.

Key words: vaginal recurrence, high-dose-rate brachytherapy, interstitial brachytherapy, image-guided adaptive brachytherapy, post-operative radiotherapy.

\section{Purpose}

Post-operative pelvic irradiation in patients with cervical cancer has been reported to reduce pelvic recurrence and improve survival [1, 2]. However, the incidences of pelvic and vaginal recurrences in the radiation field are $8.7-21.6 \%$ and $2.2 \%$, respectively [1-3], and there is no standard treatment for previously irradiated pelvic recurrence. Several reports have indicated that re-irradiation with interstitial brachytherapy (ISBT) results in a local control rate of $36.3-76.9 \%$. Late adverse events of grade 3 or higher were observed in $7.4-25.0 \%$ of gynecological cancer patients, mainly comprising cervical cancer patients who were previously treated with defin- itive radiotherapy [4-6]. However, there are few reports on re-irradiation in uterine cancer patients with vaginal recurrence after post-operative pelvic irradiation. Furthermore, while total dose administered is lower in patients with vaginal recurrence after post-operative pelvic irradiation than in patients undergoing definitive radiotherapy (including external beam radiotherapy [EBRT] and brachytherapy) as initial treatment, brachytherapy generally delivers a higher dose to organs at risk (OARs; namely the bladder, rectum, sigmoid colon, and small intestine) in patients with vaginal recurrence after postoperative radiotherapy.

Thus, this study aimed to evaluate the doses for OARs and outcomes of re-irradiation with ISBT in uterine can- 
cer patients with vaginal recurrence after post-operative pelvic irradiation.

\section{Material and methods}

\section{Study design and patients}

Overall, 27 gynecological cancer patients with local recurrence after prior radiotherapy, including definitive radiotherapy (comprising EBRT and brachytherapy) and post-operative pelvic irradiation (comprising EBRT and/ or brachytherapy), received re-irradiation with highdose-rate (HDR) ISBT between January, 2012 and December, 2016 at the Tsukuba University Hospital. In this study, we included six uterine cancer patients who met the following criteria: 1 . Post-operative pelvic irradiation performed as initial radiotherapy; 2 . Initial radiotherapy did not include brachytherapy; and 3. No recurrence other than vaginal recurrence. Protocol was approved by the institutional review board of Tsukuba University Hospital (IRB No. H30-338), and requirement for informed consent was waived because of the retrospective nature of the study.

\section{Radiotherapy}

Previous whole-pelvis EBRT after initial surgery was delivered using a three-dimensional conformal technique (3D-CRT) or intensity-modulated radiotherapy (IMRT) using a linear accelerator photon energy higher than $10 \mathrm{MV}$. Whole-pelvis EBRT was administered at a dose of 45.0-50.4 Gy in 25-28 fractions. Concurrent chemoradiotherapy with weekly cisplatin was administered to cervical cancer patients with tumors of pathological stage $\geq \mathrm{T} 2 \mathrm{~b}$ and/or tumors of pathological lymph node status N1. Whole-pelvis EBRT was principally applied within 8 weeks post-operatively.

All patients underwent re-irradiation with HDRISBT. Brachytherapy was performed using an iridium-192 ( ${ }^{192} \mathrm{Ir}$ ) remote afterloading system (MicroSelectron HDR-TM; Nucletron, Veenendaal, The Netherlands). For all patients, planning was based on computed tomography (CT) images of $2.5-\mathrm{mm}$ slice thickness, and dose calculations were performed using Oncentra (Nucletron, Veenendaal; The Netherlands) without heterogeneity correction, in accordance with the TG-43 protocol. Martinez universal perineal interstitial template was used during ISBT. Stainless and/or plastic needles were inserted under CT guidance under spinal anesthesia and epidural anesthesia, and irradiation was performed twice a day for 3-4 days. Clinical target volume (CTV) included only macroscopic tumors identified on gynecological examination and based on $\mathrm{CT}$ and/or T2-weighted magnetic resonance imaging (MRI). An ISBT dose of 38-42 Gy in 6-7 fractions for 3-4 days was prescribed for CTV (Figure 1 ), and CTV $\mathrm{D}_{90}$ was evaluated.
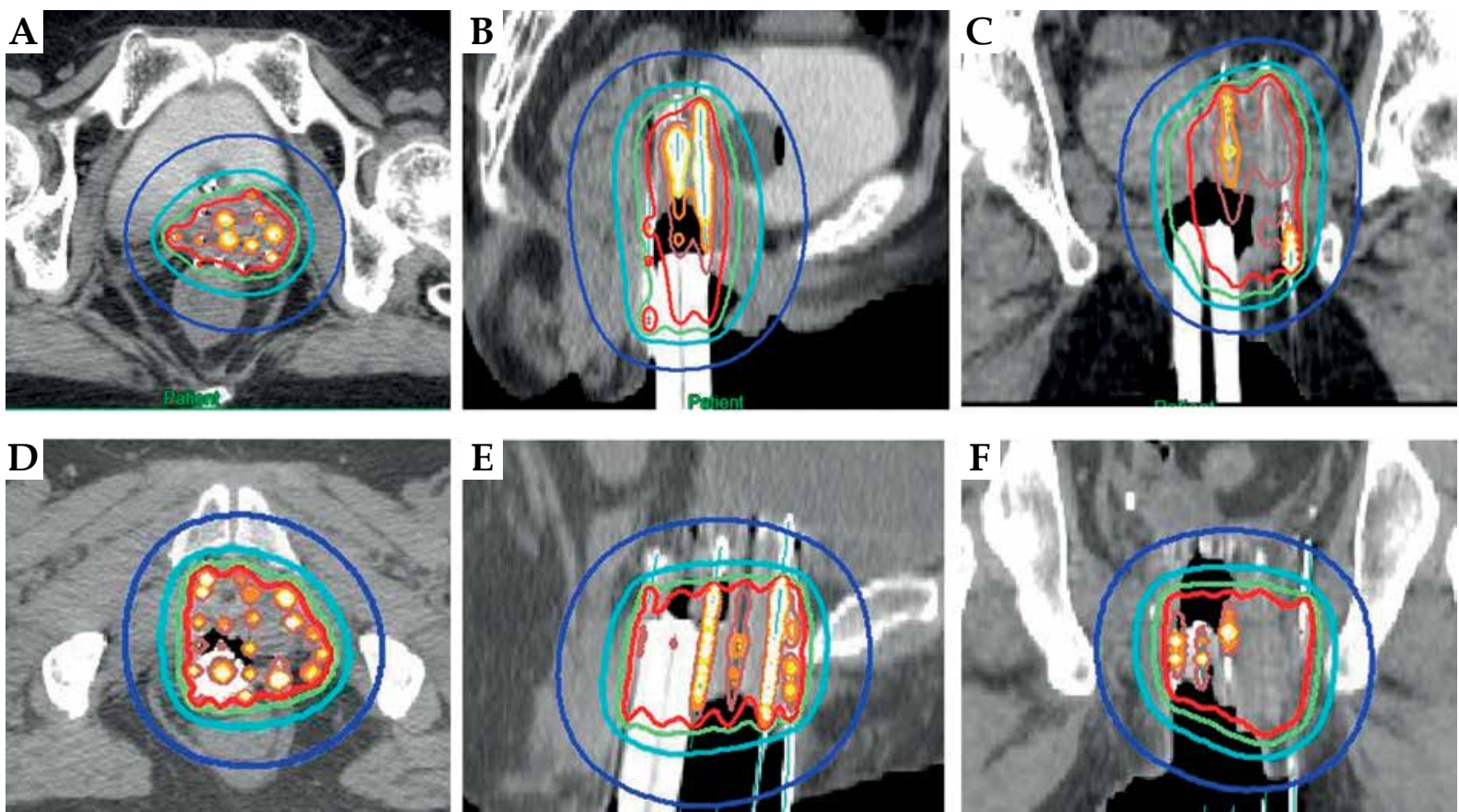

Fig. 1. Dose distribution for ISBT. Axial (A), sagittal (B), and coronal sections (C) for the vaginal recurrence at the vaginal stump after post-operative irradiation. Axial (D), sagittal (E), and coronal sections (F) for the vaginal recurrence in the vaginal wall after post-operative irradiation. For vaginal recurrence at the vaginal stump, 13 stainless steel needles were inserted using Martinez universal perineal interstitial template, and a radiation dose of $6 \mathrm{~Gy}$ per fraction was prescribed for CTV (A-C). For vaginal recurrence in the vaginal wall, 18 stainless steel needles and five plastic needles were inserted using Martinez universal perineal interstitial template, and a radiation dose of 6 Gy per fraction was prescribed for CTV (D-F). Isodose line: blue line, $25 \%$ of the prescribed dose; light blue line, $50 \%$; green line, $75 \%$; red line, $100 \%$; brown line, $150 \%$; orange line, $200 \%$; yellow line, $250 \%$; white line, $300 \%$. ISBT - interstitial brachytherapy; CTV - clinical target volume 


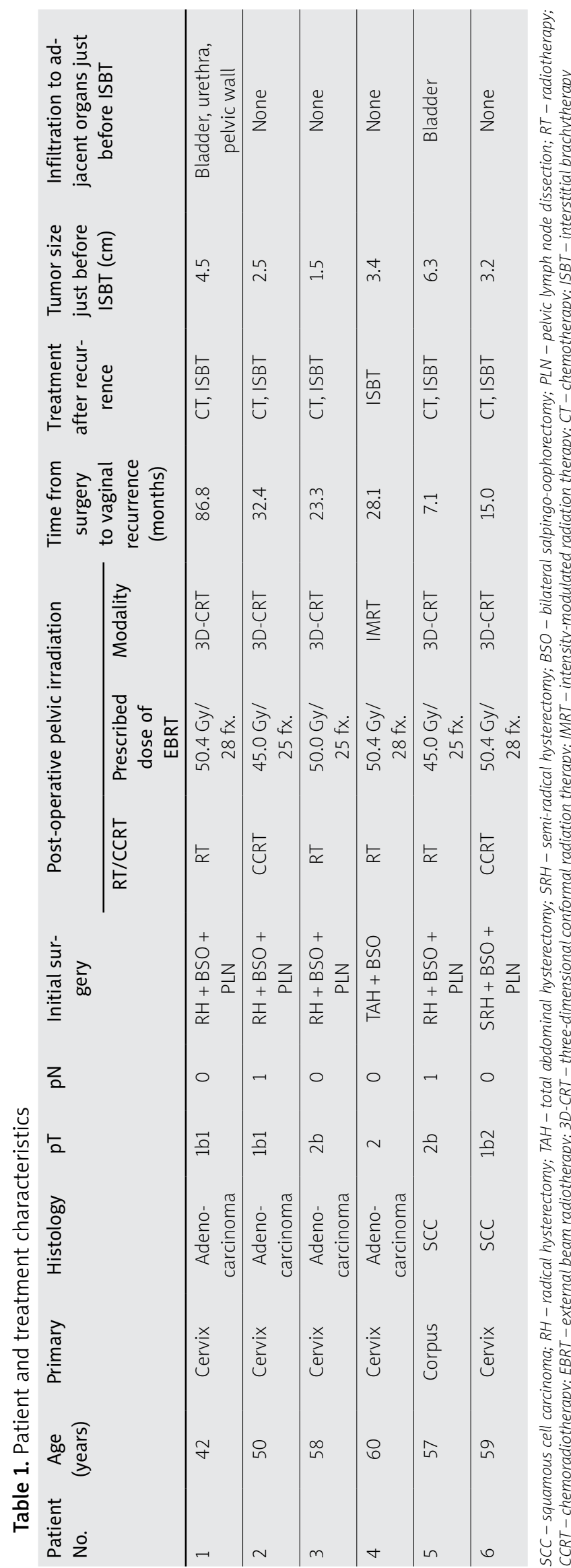

\section{Follow-up}

Treatment responses were assessed 2-3 months after HDR-ISBT completion based on response evaluation criteria in solid tumors, using histopathology and/or MRI [7]. Late adverse events were graded based on the National Cancer Institute's common terminology criteria for adverse events version 4.0. Follow-up visits comprised gynecological examination, cytology, blood tests, and imaging using CT or MRI every 2-6 months for 5 years.

\section{Statistical analysis}

For dose summation of brachytherapy and post-operative pelvic irradiation, equivalent dose in 2 Gy fractions $\left(\mathrm{EQD}_{2}\right)$ was calculated based on a linear-quadratic model [8]. $\alpha / \beta$ ratio was assumed to be 3 Gy when calculating dose-volume parameters of OARs (i.e., $\mathrm{D}_{0.1 \mathrm{cc}}$ and $\mathrm{D}_{2 \mathrm{cc}}$ ) [9].

Times to local recurrence, distant metastasis, and late adverse events were defined as periods from the completion of ISBT to the diagnosis of local recurrence, diagnosis of distant metastasis, and occurrence of adverse events, respectively. Follow-up duration was defined as the period from the completion of ISBT to the last medical examination.

\section{Results}

Patients and treatment characteristics are presented in Table 1. The median age of the patients was 57.5 years (range, 42-60 years). Five of the six patients had cervical cancer, while one had endometrial cancer. Post-operative pelvic irradiation was delivered to five and one patients using 3D-CRT and IMRT, respectively. The median time from initial surgery to vaginal recurrence was 25.7 months (range, 7.1-86.8 months). The median time from the completion of pelvic irradiation to ISBT initiation was 24.1 months (range, 9.0-104.5 months). The median tumor size, as measured using MRI just before ISBT, was $3.3 \mathrm{~cm}$ (range, $1.5-6.3 \mathrm{~cm}$ ).

Table 2 summarizes CTV volumes, $\mathrm{D}_{90}$ for CTV and $\mathrm{D}_{2 \mathrm{cc}}$ for OARs at ISBT, and total $\mathrm{EQD}_{2}$ of $\mathrm{D}_{2 \mathrm{cc}}$ for OARs (including EBRT and ISBT). The mean CTV volume was 49.5 cc. The mean doses per fraction of ISBT for CTV $D_{90}$, $\mathrm{D}_{2 \mathrm{cc}}$ for the bladder, and $\mathrm{D}_{2 \mathrm{cc}}$ for the rectum were $6.1 \mathrm{~Gy}$, $4.4 \mathrm{~Gy}$, and $3.8 \mathrm{~Gy}$, respectively. $\mathrm{D}_{2 \mathrm{cc}}$ for the sigmoid for patient number 1,4 , and 5 were $<0.1$ Gy because of the distance between the vaginal tumor and the sigmoid. The mean total $\mathrm{EQD}_{2}$, including EBRT and ISBT, for $\mathrm{D}_{2 \mathrm{cc}}$ for the bladder, sigmoid, and rectum were $92.1 \mathrm{~Gy}, 50.4 \mathrm{~Gy}$, and $81.6 \mathrm{~Gy}$, respectively.

Outcomes and adverse events are presented in Table 3. The median follow-up duration was 53.3 months (range, 17.7-71.8 months). A complete response (CR) was achieved in five of the six patients. Local recurrence was observed in two patients who experienced vaginal recurrence infiltrating the adjacent organs, such as the bladder and the rectum. For patient number 1, local recurrence, which was located above the vaginal stump, was observed at 26.5 months after re-irradiation with ISBT; the tumor grew rapidly, resulting in an enterovaginal fistula. The total $\mathrm{EQD}_{2}$ for $\mathrm{D}_{0.1 \mathrm{cc}}$ of the bladder and rectum were 
Table 2. Doses for clinical target volumes (CTVs) and organs at risk (OARs)

\begin{tabular}{|c|c|c|c|c|c|c|c|c|c|}
\hline \multirow{2}{*}{$\begin{array}{l}\text { Patient } \\
\text { No. }\end{array}$} & \multirow{2}{*}{$\begin{array}{l}\text { Prescribed dose } \\
\text { of ISBT }\end{array}$} & \multirow{2}{*}{$\begin{array}{c}\text { CTV } \\
\text { volume }\end{array}$} & \multicolumn{4}{|c|}{ Dose per fraction of ISBT for CTVs and OARs } & \multicolumn{3}{|c|}{ Total EQD 2 for OARs (EBRT + ISBT) } \\
\hline & & & $\begin{array}{c}\text { CTV D } D_{90} \\
(G y)\end{array}$ & $\begin{array}{c}\text { Bladder } \\
D_{2 c c}(G y)\end{array}$ & $\begin{array}{l}\text { Sigmoid } \\
D_{2 c c}(G y)\end{array}$ & $\begin{array}{l}\text { Rectum } \\
D_{2 c c}(G y)\end{array}$ & $\begin{array}{c}\text { Bladder } \\
D_{2 c c}(G y)\end{array}$ & $\begin{array}{l}\text { Sigmoid } \\
D_{2 c c}(G y)\end{array}$ & $\begin{array}{c}\text { Rectum } \\
D_{2 c c}(G y)\end{array}$ \\
\hline 1 & $38.0 \mathrm{~Gy} / 6 \mathrm{fx}$ & $42.4 \mathrm{cc}$ & 5.8 & 6.1 & N.A. & 3.8 & 114.1 & 48.4 & 79.8 \\
\hline 2 & $42.0 \mathrm{~Gy} / 7 \mathrm{fx}$ & $28.3 \mathrm{cc}$ & 6.0 & 4.8 & 1.2 & 3.4 & 94.9 & 50.3 & 73.7 \\
\hline 3 & $42.0 \mathrm{~Gy} / 7 \mathrm{fx}$ & $10.8 c c$ & 6.8 & 3.7 & 1.4 & 3.9 & 84.0 & 58.9 & 87.2 \\
\hline 4 & $42.0 \mathrm{~Gy} / 7 \mathrm{fx}$ & $35.4 \mathrm{cc}$ & 6.4 & 1.5 & N.A. & 3.5 & 58.0 & 48.4 & 80.7 \\
\hline 5 & $42.0 \mathrm{~Gy} / 7 \mathrm{fx}$ & $144.2 \mathrm{cc}$ & 5.8 & 6.1 & N.A. & 3.8 & 108.9 & 43.2 & 80.0 \\
\hline 6 & $42.0 \mathrm{~Gy} / 7 \mathrm{fx}$ & $35.7 c c$ & 5.6 & 4.3 & 0.9 & 4.1 & 92.8 & 53.4 & 88.5 \\
\hline
\end{tabular}

CTV - clinical target volume; OAR - organ at risk; ISBT - interstitial brachytherapy; $E Q D_{2}$ - equivalent dose in 2 Gy fractions; EBRT - external beam radiotherapy; N.A. - not applicable

Table 3. Outcomes and adverse events

\begin{tabular}{|c|c|c|c|c|c|c|}
\hline & \multicolumn{6}{|c|}{ Patient No. } \\
\hline & 1 & 2 & 3 & 4 & 5 & 6 \\
\hline $\begin{array}{l}\text { Complete re- } \\
\text { sponse }\end{array}$ & Yes & Yes & Yes & Yes & No & Yes \\
\hline Local control & No & Yes & Yes & Yes & No & Yes \\
\hline $\begin{array}{l}\text { Time to local } \\
\text { recurrence } \\
\text { (months) }\end{array}$ & 26.5 & N.A. & N.A. & N.A. & 0.0 & N.A. \\
\hline $\begin{array}{l}\text { Distant metas- } \\
\text { tasis }\end{array}$ & Yes & Yes & No & No & No & No \\
\hline $\begin{array}{l}\text { Time to distant } \\
\text { metastasis } \\
\text { (months) }\end{array}$ & 26.5 & 9.5 & N.A. & N.A. & N.A. & N.A. \\
\hline $\begin{array}{l}\text { Site of distant } \\
\text { metastasis }\end{array}$ & Bone & PALN & N.A. & N.A. & N.A. & N.A. \\
\hline Alive/dead & Dead & Alive & Alive & Alive & Dead & Alive \\
\hline Adverse events & $\begin{array}{l}\text { Entero-vaginal } \\
\text { fistula gr. } 4\end{array}$ & Proctitis gr. 2 & Proctitis gr. 2 & None & $\begin{array}{l}\text { Vesico-vaginal } \\
\text { and recto-vagi- } \\
\text { nal fistulas gr. } 4\end{array}$ & None \\
\hline $\begin{array}{l}\text { Time to ad- } \\
\text { verse events } \\
\text { (months) }\end{array}$ & 27.4 & 14.8 & 35.4 & N.A. & 9.4 & N.A. \\
\hline $\begin{array}{l}\text { Follow-up period } \\
\text { (months) }\end{array}$ & 31.0 & 71.8 & 69.5 & 59.2 & 17.7 & 47.4 \\
\hline
\end{tabular}

PLAN - para-aortic lymph node; gr. - grade; N.A. - not applicable

169.0 Gy and 91.9 Gy, respectively. For patient number 5, CR was not achieved, and vaginal recurrence, which was located above the vaginal stump, had re-growth at 7.4 months after re-irradiation with ISBT, and led to the formation of vesico-vaginal and recto-vaginal fistulas at 2 months after re-growth of vaginal recurrence. The total $\mathrm{EQD}_{2}$ for $\mathrm{D}_{0.1 \mathrm{cc}}$ of the bladder and rectum were $141.1 \mathrm{~Gy}$ and $94.2 \mathrm{~Gy}$, respectively. These two patients were managed by colostomy due to the fistulas. At the time of analysis, four of the six patients were alive. Grade 2 late rectal complications occurred in two patients (patient number 2 and patient number 3; 33.3\%). In particular, one patient received iron for mild anemia secondary to a persistent small amount of rectal bleeding, while the other patient underwent argon plasma coagulation for mild rectal bleeding that did not cause anemia. No late compli- cations of grade $\geq 3$ were observed in four alive patients. Grade 4 complications were observed in patients number 1 and 5 who had local recurrence infiltrating to adjacent organs (i.e., the bladder and rectum) after re-irradiation with ISBT.

\section{Discussion}

For vaginal recurrence in uterine cancer patients without previous pelvic irradiation, definitive radiotherapy (including brachytherapy) has been the standard treatment, with a good local control rate of approximately 95\% [10-12]. In guidelines reported by the American Brachytherapy Society (ABS), the use of ISBT was recommended for vaginal tumors $>5 \mathrm{~mm}$ to deliver a sufficient dose to tumor while sparing OARs [13]. However, there 
is no standard treatment for patients with vaginal recurrence who have undergone pelvic irradiation previously.

Chemotherapy is not a definitive treatment option for gynecological cancer patients with pelvic recurrence primarily treated with surgery $[14,15]$. Furthermore, the response rates of chemotherapy for recurrence in an irradiated field (30-33\%) were lower than those for recurrence outside an irradiated field (60-75\%) [16-18]. Additionally, it has been reported that pelvic exenteration leads to overall survival rates of $26.7-65.8 \%$ in cervical cancer patients with local recurrence after radiotherapy [19-22]. Shingleton et al. showed that pelvic control after pelvic exenteration was achieved in 99 (69.2\%) of the 143 cervical cancer patients with local recurrence after pelvic irradiation [22]. However, mortality and complications caused by pelvic exenteration were very high (4.8-6.3\% and $56.9-65.3 \%$, respectively) [19, 20, 22].

Recent reports have shown that re-irradiation with ISBT could lead to 2-year survival, with local control rates of $54.7-78.0 \%$ and $50.0-71.4 \%$, respectively, in gynecological cancer patients who have received pelvic irradiation previously. The incidence rate of grade $\geq 3$ late complications, including bowel obstruction, recto-vaginal fistula, and vesico-vaginal fistula was 7.7-25.0\% [4-6, 23] When comparing to pelvic exenteration, re-irradiation with ISBT could also achieve a lower incidence of grade $\geq 3$ late complications. Xiang et al. reported their results of re-irradiation with ISBT for vaginal recurrence after definitive radiotherapy, with EBRT and brachytherapy, in cervical cancer patients [23]. Other recent reports have included patients receiving definitive radiotherapy and those treated with post-operative radiotherapy as initial treatment $[5,6]$. However, few studies have included only patients with vaginal recurrence after post-operative pelvic irradiation [24]. In our study, four (66.7\%) of the six patients who previously received post-operative pelvic irradiation achieved local control, and the median survival period was 53.3 months (range, 17.7-71.8 months). In the remaining two patients with local recurrence, fistulas were observed after re-irradiation with ISBT, and the total $\mathrm{EQD}_{2}$ for $\mathrm{D}_{0.1 \mathrm{cc}}$ of the bladder and rectum were $>140$ Gy and > 91 Gy, respectively, which was extremely high. Therefore, there was a possibility that the fistulas were caused by radiotherapy. However, in both patients, local recurrences were diagnosed by cytological analysis after re-irradiation with ISBT (one patient; CR not achieved), and CT revealed that the fistulas had been formed by growing recurrences. Thus, grade $\geq 3$ late complications were not observed in four alive patients, who achieved local control after re-irradiation with ISBT, at the time of analysis.

A prospective multi-institutional European study on MRI-guided brachytherapy in patients with locally advanced cervical cancer showed that grade 1, 2, 3, and 4 late rectal complications were observed in $20.1 \%, 6.0 \%$, $1.6 \%$, and $0.1 \%$ of the patients, respectively. $\mathrm{EQD}_{2}$ for $\mathrm{D}_{2 \mathrm{cc}}$ for a $10 \%$ probability of overall rectal grade $\geq 2$ toxicity was $69.5 \mathrm{~Gy}$, with more severe rectal complications and higher frequencies occurring with a $D_{2 c c}$ value of $\geq 75$ Gy [25]. Liu et al. reported no serious complications in cervical cancer patients treated with ISBT for vaginal recurrence after post-operative pelvic irradiation with a to- tal dose of 45 Gy in 25 fractions, although the observation period was short [24]. In their study, mean total $\mathrm{EQD}_{2}$ for the bladder, sigmoid, and rectum $\mathrm{D}_{2 \mathrm{cc}}$ for dose summation of ISBT and post-operative pelvic irradiation were $85.6 \pm 5.8 \mathrm{~Gy}, 69.6 \pm 5.9 \mathrm{~Gy}$, and 71.6 $\pm 6.4 \mathrm{~Gy}$, respectively. In our study, the mean total $\mathrm{EQD}_{2}$ for the bladder, sigmoid, and rectum $\mathrm{D}_{2 \mathrm{cc}}$ were $92.1 \pm 20.5 \mathrm{~Gy}, 50.4 \pm 19.4 \mathrm{~Gy}$, and $81.6 \pm 10.9 \mathrm{~Gy}$, respectively. The total $\mathrm{EQD}_{2}$ for $\mathrm{D}_{2 \mathrm{cc}}$ for the bladder and rectum in four and five of the six patients, respectively, exceeded the dose recommended by the ABS (bladder, $\leq 90 \mathrm{~Gy}$; rectum and sigmoid, $\leq 75 \mathrm{~Gy}$ ) [13]. In two patients, who were managed by colostomy due to fistula caused by local recurrence infiltrating to adjacent organs after re-irradiation with ISBT, grade 4 late complications were observed. However, in these two patients, $\mathrm{EQD}_{2}$ of $\mathrm{D}_{0.1 \mathrm{cc}}$ for the bladder and rectum were $>140$ Gy and >91 Gy, respectively. Therefore, the possibility of fistula caused by radiation could not be ruled out. At least, in four alive patients without local recurrence after re-irradiation with ISBT, it could be mentioned that grade $\geq 3$ late complications were not observed during analysis (median follow-up duration, 64.4 months; range, 47.4-71.8 months). Ang et al. suggested the possibility that occult injuries induced by irradiation in rhesus monkeys substantially resolved within 2 years after radiotherapy [26]. In our study, a break between the completion of pelvic irradiation and ISBT initiation (median, 24.1 months; range, 9.0-104.5 months) may be associated with the low incidence of severe late complications.

In patients treated with pelvic exenteration for pelvic recurrence after pelvic irradiation, poor survival was associated with large recurrent tumors $(\geq 3 \mathrm{~cm})$, recurrence with pelvic wall infiltration, and short interval between completion of initial treatment and pelvic recurrence ( $\leq 1$ year) [20-22]. Moreover, in patients undergoing re-irradiation with ISBT for pelvic recurrence after pelvic irradiation, a large recurrent tumor $(\geq 4 \mathrm{~cm})$ was a poor prognostic factor for survival and local control, and a short interval between the completion of initial treatment and pelvic recurrence ( $\leq 6$ months) was associated with poor survival $[5,23]$. In our study, local recurrence and subsequent death were observed in two patients; one had vaginal recurrence with a large tumor $(4.5 \mathrm{~cm})$ and pelvic wall infiltration, while the other had a large tumor $(6.3 \mathrm{~cm})$. The remaining four patients without local recurrence at the time of analysis had small vaginal recurrence $<4.0 \mathrm{~cm}$ without pelvic wall infiltration. These results might encourage regular gynecological examination for early detection of vaginal recurrence and consideration of re-irradiation with ISBT as the first-line treatment for vaginal recurrence after post-operative pelvic irradiation.

The limitations of our study included its' retrospective design and small group of the patients. Moreover, bias was present in the patients' clinical backgrounds. Thus, further multicenter studies that involve a larger patient population with uniform clinical backgrounds are required to verify our findings. However, the outcomes of this study suggested that re-irradiation with ISBT could be a treatment option for small vaginal recurrence after post-operative pelvic irradiation. 


\section{Conclusions}

Re-irradiation with ISBT achieved good local control and a low-rate of late complications after post-operative irradiation among patients with small vaginal recurrence $<4.0 \mathrm{~cm}$ without pelvic wall infiltration. Although the total doses of OARs, which were delivered by post-operative pelvic irradiation and ISBT, exceeded the doses recommended by the ABS for almost all patients, a long interval between completion of pelvic irradiation and ISBT initiation may be associated with a low incidence of severe late complications. Re-irradiation with ISBT may be an effective treatment strategy for gynecological cancer patients with small vaginal recurrence after post-operative pelvic irradiation.

\section{Acknowledgments}

This work was partially supported by Grants-in-Aid for Scientific Research (B) (19 H 03596 ) from the Ministry of Education, Culture, Sports, Science, and Technology of Japan.

\section{Disclosure}

The authors report no conflict of interest.

\section{References}

1. Rotman M, Sedlis A, Piedmonte MR et al. A phase III randomized trial of postoperative pelvic irradiation in stage IB cervical carcinoma with poor prognostic features: follow-up of a gynecologic oncology group study. Int J Radiat Oncol Biol Phys 2006; 65: 169-176.

2. Peters WA 3rd, Liu PY, Barrett RJ 2nd et al. Concurrent chemotherapy and pelvic radiation therapy compared with pelvic radiation therapy alone as adjuvant therapy after radical surgery in high-risk early-stage cancer of the cervix. J Clin Oncol 2000; 18: 1606-1613.

3. Monk BJ, Wang J, Im S et al. Rethinking the use of radiation and chemotherapy after radical hysterectomy: a clinical-pathologic analysis of a Gynecologic Oncology Group/ Southwest Oncology Group/Radiation Therapy Oncology Group trial. Gynecol Oncol 2005; 96: 721-728.

4. Raziee H, D'Souza D, Velker V et al. Salvage re-irradiation with single-modality interstitial brachytherapy for the treatment of recurrent gynaecological tumours in the pelvis: a multi-institutional study. Clin Oncol (R Coll Radiol) 2020; 32: 43-51.

5. Mabuchi S, Takahashi R, Isohashi F et al. Reirradiation using high-dose-rate interstitial brachytherapy for locally recurrent cervical cancer: a single institutional experience. Int J Gynecol Cancer 2014; 24: 141-148.

6. Martinez-Monge R, Cambeiro M, Rodríguez-Ruiz ME et al. Phase II trial of image-based high-dose-rate interstitial brachytherapy for previously irradiated gynecologic cancer. Brachytherapy 2014; 13: 219-224.

7. Eisenhauer EA, Therasse P, Bogaerts J et al. New response evaluation criteria in solid tumours: revised RECIST guideline (version 1.1). Eur J Cancer 2009; 45: 228-247.

8. Dale RG. The application of the linear-quadratic dose-effect equation to fractionated and protracted radiotherapy. Br J Radiol 1985; 58: 515-528.

9. Williams MV, Denekamp J, Fowler JF. A review of alpha/ beta ratios for experimental tumors: implications for clinical studies of altered fractionation. Int J Radiat Oncol Biol Phys 1985; 11: 87-96.
10. Lee LJ, Damato AL, Viswanathan AN. Clinical outcomes following 3D image-guided brachytherapy for vaginal recurrence of endometrial cancer. Gynecol Oncol 2013; 131: 586-592.

11. Fokdal L, Ørtoft G, Hansen ES et al. Toward four-dimensional image-guided adaptive brachytherapy in locally recurrent endometrial cancer. Brachytherapy 2014; 13: 554-561.

12. Vargo JA, Kim H, Houser CJ et al. Definitive salvage for vaginal recurrence of endometria cancer: the impact of modern intensity-modulated-radiotherapy with image-based HDR brachytherapy and the interplay of the PORTEC 1 risk stratification. Radiother Oncol 2014; 113: 126-131.

13. BeriwalS, Demanes DJ, Erickson B et al. American Brachytherapy Society consensus guidelines for interstitial brachytherapy for vaginal cancer. Brachytherapy 2012; 11: 68-75.

14. Koh WJ, Abu-Rustum NR, Bean $\mathrm{S}$ et al. Cervical cancer, version 3.2019, NCCN clinical practice guidelines in oncology. J Natl Compr Canc Netw 2019; 17: 64-84.

15. Koh WJ, Abu-Rustum NR, Bean S et al. Uterine neoplasms, version 1.2018, NCCN clinical practice guidelines in oncology. J Natl Compr Canc Netw 2018; 16: 170-199.

16. Benjapibal M, Thirapakawong $C$, Leelaphatanadit $C$ et al. A pilot phase II study of capecitabine plus cisplatin in the treatment of recurrent carcinoma of the uterine cervix. Oncology 2007; 72: 33-38.

17. Pectasides D, Fountzilas G, Papaxoinis G et al. Carboplatin and paclitaxel in metastatic or recurrent cervical cancer. Int J Gynecol Cancer 2009; 19: 777-781.

18. Kosmas C, Mylonakis N, Tsakonas G et al. Evaluation of the paclitaxel-ifosfamide-cisplatin (TIP) combination in relapse and/or metastatic cervical cancer. Br J Cancer 2009; 101: 1059-1065.

19. Marnitz S, Köhler C, Müller M et al. Indications for primary and secondary exenterations in patients with cervical cancer. Gynecol Oncol 2006; 103: 1023-1030.

20. Terán-Porcayo MA, Zeichnner-Gancz I, del-Catillo RA et al. Pelvic exenteration for recurrent or persistent cervical cancer: experience of five years at the National Cancer Institute in Mexico. Med Oncol 2006; 23: 219-223.

21. Chiantera V, Rossi M, De Iaco P et al. Survival after curative pelvic exenteration for primary or recurrent cervical cancer: a retrospective multicentric study of 167 patients. Int J Gynecol Cancer 2014; 24: 916-922.

22. Shingleton HM, Soong SJ, Gelder MS et al. Clinical and histopathologic factors predicting recurrence and survival after pelvic exenteration for cancer of the cervix. Obstet Gynecol 1989; 73: 1027-1034.

23. Xiang EW, Shumo C, Yaqin D et al. Treatment of late recurrent vaginal malignancy after initial radiotherapy for carcinoma of the cervix: an analysis of 73 cases. Gynecol Oncol 1998; 69: 125-129.

24. Liu ZS, Guo J, Zhao YZ et al. Salvage interstitial brachytherapy based on computed tomography for recurrent cervical cancer after radical hysterectomy and adjuvant radiation therapy: case presentations and introduction of the technique. J Contemp Brachytherapy 2016; 8: 415-421.

25. Mazeron R, Fokdal LU, Kirchheiner K et al. Dose-volume effect relationships for late rectal morbidity in patients treated with chemoradiation and MRI-guided adaptive brachytherapy for locally advanced cervical cancer: results from the prospective multicenter EMBRACE study. Radiother Oncol 2016; 120: 412-419.

26. Ang KK, Jiang GL, Feng Y et al. Extent and kinetics of recovery of occult spinal cord inury. Int J Radiat Oncol Biol Phys 2001; 50: 1013-1020. 